\title{
Identifying risk factors of anemia among women of reproductive age in Rwanda - a cross-sectional study using secondary data from the Rwanda demographic and health survey 2014/2015
}

Dieudonne Hakizimana ${ }^{1 *}$ (D), Marie Paul Nisingizwe ${ }^{2}$, Jenae Logan $^{1}$ and Rex Wong ${ }^{1,3}$

\begin{abstract}
Background: Anemia among Women of Reproductive Age (WRA) continues to be among the major public health problems in many developing countries, including Rwanda, where it increased in prevalence between the 2015 and 2010 Rwanda Demographic and Health Survey (RDHS) reports. A thorough understanding of its risk factors is necessary to design better interventions. However, to the best of our knowledge, no study has been conducted in Rwanda on a nationally representative sample to assess factors associated with anemia among WRA. Accordingly, this study was conducted to address such gap.
\end{abstract}

Methods: A quantitative, cross-sectional study was conducted using data from the RDHS 2014-2015. The study population consisted of 6680 WRA who were tested for anemia during the survey. Anemia was defined as having a hemoglobin level equal to or below $10.9 \mathrm{~g} / \mathrm{dl}$ for a pregnant woman, and hemoglobin level equal to or below 11.9 $\mathrm{g} / \mathrm{dl}$ for a non-pregnant woman. Pearson's chi-squared test and multiple logistic regression were conducted for bivariate and multivariable analysis, respectively.

Results: The prevalence of anemia among WRA was 19.2\% (95\% Cl: 18.0-20.5). Four factors were found to be associated with lower odds of anemia, including being obese (OR: 0.61,95\% Cl: 0.40-0.91), being in the rich category (OR: $0.74,95 \% \mathrm{Cl}: 0.63-0.87$ ), sleeping under a mosquito net (OR: $0.85,95 \% \mathrm{Cl}: 0.74-0.98)$, and using hormonal contraceptives (OR: $0.61,95 \%$ Cl: 0.50-0.73). Five factors were associated with higher odds of anemia, including being underweight (OR: 1.39, 95\% Cl: 1.09-1.78), using an intrauterine device (OR: 1.98, 95\% Cl: 1.05-3.75), being separated or widowed (OR: 1.35, 95\% Cl: 1.09-1.67), and living in the Southern province (OR: 1.45, 95\% Cl: 1.11-1.89) or in the Eastern province (OR: 1.41, 95\% Cl: 1.06-1.88).

Conclusion: Anemia continues to pose public health challenges; novel public health interventions should consider geographic variations in anemia risk, seek to improve women's economic statuses, and strengthen iron supplementation especially for Intrauterine device users. Additionally, given the association between anemia and malaria, interventions to prevent malaria should be enhanced.

Keywords: Anemia, Women of reproductive age, Malaria, Prevalence, Associated factors

\footnotetext{
* Correspondence: dieudonnehakiziman@gmail.com; dhakizimana@ughe.org

'Department of Global Health Delivery, University of Global Health Equity,

Kigali, Rwanda

Full list of author information is available at the end of the article
}

(c) The Author(s). 2019 Open Access This article is distributed under the terms of the Creative Commons Attribution 4.0 International License (http://creativecommons.org/licenses/by/4.0/), which permits unrestricted use, distribution, and reproduction in any medium, provided you give appropriate credit to the original author(s) and the source, provide a link to the Creative Commons license, and indicate if changes were made. The Creative Commons Public Domain Dedication waiver (http://creativecommons.org/publicdomain/zero/1.0/) applies to the data made available in this article, unless otherwise stated. 


\section{Background}

Anemia is a significant public health problem affecting around 1.93 billion people worldwide [1]. It affects $29.4 \%$ of Women of Reproductive Age (WRA) and 38.2\% of pregnant women [2]. Anemia among pregnant women is associated with increased risks of maternal mortality, preterm birth, perinatal mortality and neonatal mortality, as well as low birth weight and anemia for the child [3-6]. Anemia affects cognitive development in children and reduces women's work abilities, and it is ultimately associated with increased healthcare expenditures $[2,7,8]$.

Anemia disproportionally affects developing countries, with Asian and Sub-Saharan African countries bearing $89 \%$ of the anemia burden [1]. More than $38 \%$ of all WRA in the African region are anemic [2], with significant variations between countries; in some African countries, the prevalence of anemia among WRA is higher than 50\% [9].

Rwanda has made progress in improving maternal health, but anemia among WRA remains a burden. The 2014-15 Demographic and Health Survey (DHS) report estimated the prevalence of anemia among WRA in Rwanda to be $19.2 \%$ [10]. Although this is considered a mild public health problem according to $\mathrm{WHO}$ criteria [11], the prevalence had risen from 17\% in 2010 [10]. Moreover, 11 of 30 districts in Rwanda reported much higher prevalence than the national average; with some districts even reporting prevalence of more than $30 \%[10,12,13]$.

The increase in national prevalence and disparity across subgroups and regions suggests that, if unaddressed, the problem will grow despite current efforts made to improve the health of the population. Understanding the risk factors for anemia is crucial for the development of innovative and evidence-based interventions to reduce its prevalence nationwide. However, the few studies conducted in Rwanda to identify risk factors for anemia were restricted to children or only a subset of WRA such as women living with Human Immunodeficiency Virus infection or young women (18-27 years old); such studies lacked national representation and also had inconsistent and conflicting results [13-18]. Accordingly, this study was conducted to understand the variations of anemia among WRA in Rwanda according to their background characteristics and to identify associated risk factors using nationally representative Rwanda Demographic and Health Survey (RDHS) data in order help inform the design of specific interventions to reduce the prevalence of anemia among WRA.

\section{Methods}

\section{Study setting}

Rwanda is a land-locked African country with an estimated population of $10,515,973$ in 2012, of which $51.8 \%$ were female. Around $48.55 \%$ of all female are women of reproductive age, between 15 and 49 years of age [19]. The country is characterized by rapid population growth
(2.6\% annually) and a GDP per capita of 702.84 USD $[19,20]$. Rwanda has demonstrated marked improvement in maternal health over the past 20 years [21]. In 2015, the Total Fertility Rate was 4.2 (down from 6.1 in 2005), and the maternal mortality rate was 210 per 100 , 000 live births, reduced from 1071 in 2000 . Ninety-nine percent (99\%) of pregnant women attend at least one antenatal care session, and $91 \%$ delivered at health facilities. The contraceptive prevalence rate was estimated to be $53 \%$ [10].

\section{Study design}

This study used a quantitative, cross-sectional design by analysing data from the 2014-2015 Rwanda Demographic and Health Survey (RDHS).

\section{Sampling and data collection method}

The 2014-15 RDHS data were collected using standard Demographic and Health Survey (DHS) questionnaires, which were adapted by stakeholders from the Rwandan government and its partners to reflect specific social and cultural issues in Rwanda. All questionnaires were translated from English to Kinyarwanda and were pre-tested prior to actual data collection. The data collection was conducted by qualified and trained professionals with rigorous supervision. Sampling was based on the 2012 Rwanda Population and Housing Census (RPHC) which consists of a list of villages, considered enumeration areas (EAs). They were stratified by type of residence (rural or urban) in each district, and 60 sampling strata were created from all districts. A two-stage cluster sampling design was used to ensure that estimates were representative at national level. At the first stage, 492 EAs were selected from all sampling strata (113 from urban areas and 379 from rural areas), and then, a systematic sampling strategy was carried out by first listing all households in selected EAs. At the second stage, 26 households were randomly selected from each EA, resulting in 12,792 households total. Anemia was tested for in half of the households selected for the general survey, resulting in a representative sample of 6680 eligible women being tested [10]. On-site hemoglobin analysis was conducted to test for anemia using a batteryoperated portable HemoCue analyzer, and results were adjusted for altitude and for smoking status (if known) using the recommended Centers for Disease Control and Prevention (CDC) formula [10]. Our study analyzed all WRA in RDHS with anemia test results.

\section{Variables}

\section{Dependent variables}

Our dependent variable was anemia status at the time of the survey. Pregnant women with hemoglobin level equal to or below $10.9 \mathrm{~g} / \mathrm{dl}$ and non-pregnant women 
with hemoglobin level equal to or below $11.9 \mathrm{~g} / \mathrm{dl}$ were considered anemic. The other anemia operational definitions considered were mild anemia, defined by hemoglobin levels between 10.0-10.9 g/dl and 10.0-11.9 $\mathrm{g} / \mathrm{dl}$ in pregnant women and non-pregnant women, respectively; moderate anemia, defined by hemoglobin level between 7.0 and $9.9 \mathrm{~g} / \mathrm{dl}$; and severe anemia, defined by hemoglobin level less than $7.0 \mathrm{~g} / \mathrm{dl}$.

\section{Independent variables}

Based on previous literature and biological knowledge, the independent variables included in the analysis were social and demographic characteristics (age of the respondent, province of residence, rural vs. urban residence, educational attainment, economic status, union/ marital status), variables related to reproductive health and mother's health status (pregnancy status, number of children ever born from the woman, breastfeeding status, body mass index, use of family planning method). We also included variables related to access to information (frequency of reading newspaper or magazine, listening to radio, and watching television), and variables related to living conditions (including having a toilet facility in the household, main source of drinking water for the household, having a mosquito bed net for sleeping, respondent having slept under a mosquito bed net the night before the survey, and respondent considering distance to health facility as a problem).

\section{Data access and analysis}

The RDHS data were downloaded from the DHS program website in STATA format after the investigators received approval from the DHS program. The "Severe", "Moderate" and "Mild" anemia levels from the DHS was recoded into "Anemia" in our analysis; and the variable "Marital status" was regrouped into 3 levels as "Single", "Married/living together" and "Separated/widowed". Socio-economic status was consolidated from five to three categories. Other variables including contraceptive method, number of children ever born, and recent births in past years were regrouped by combining the less frequent options into one group for analysis. BMI was converted from a numeric to a categorical variable based on WHO body mass index [22]. We conducted descriptive analyses to summarize anemia status according to the independent variables. Pearson's chi-squared test was used to evaluate the association between anemia status and other independent variables. To identify factors associated with anemia among WRA in Rwanda, all variables found significant in bivariate analyses were further analyzed using multivariate analysis with backward stepwise logistic regression, after checking for collinearity. A variable was removed from the full model when it was not statistically significant at $p=0.05$. Sampling weights were included in all analyses to adjust for the effects of the stratification and cluster sampling approaches used in RDHS. Odds Ratios (OR), 95\% confidence intervals (CI) and $p$-values were reported. All data analyses were conducted using STATA version 14.2 (StataCorp Lakeway Drive College Station, Texas).

\section{Results}

\section{Socio-demographic characteristics}

A total of 6680 women were included in the analysis. The mean age was 28.6 years (SD: 9.4). Among the samples, $1386(20.7 \%)$ were 15-19 years old, 1225 (18.3\%) were 20-24 years old, and 1153 (17.3\%) were 25-29 years old. Around $24.6 \%$ (1646) of the participants were from the Eastern province, $21.6 \%$ (1442) were from the Western province, 24\% (1605) were from the Southern province, $16.3 \%$ (1088) were from the Northern province and $13.5 \%$ (899) were from Kigali city. Only $184(2.8 \%)$ had completed education above secondary level and 2863 (42.9\%) completed primary school level. More than half ( $n=3434,51.4 \%)$ of the women were married or living together with their husbands; 2622 (39.3\%) were in the poor economic category, 1249 (18.7\%) were in the middle category and 2809 (42.1\%) were in the rich category (Tables 1 and 2).

\section{Anemia status}

The mean Haemoglobin $(\mathrm{Hb})$ level across the sample, adjusted for altitude and smoking status, was $13.02 \mathrm{~g} / \mathrm{dl}$ (SD: 1.52), and the overall prevalence of anemia was 19.2\% (95\% CI: 18.0-20.5). Most cases were mild anemia, with a prevalence of $15.7 \%$ (95\% CI: 14.6-16.8); 3.4\% (95\% CI: $2.9-3.9$ ) were moderate anemia, and $0.2 \%$ (95\% CI: 0.1-0.3) were severe anemia.

The anemia prevalence was found to be higher among women aged $45-49$ years $(24.2,95 \%$ CI: $20.3-28.1)$ and 40-44 years (19.7, 95\% CI; 16.7-23.2). It was also higher in the Southern province (22.9, 95\% CI: 20.4-25.6) and in the Eastern province (21.8, 95\% CI: 18.9-25.0). It was higher among women who were in the poor category (22.5, 95\% CI; 20.6-24.5), who had no education (22.5, 95\%CI: 19.5-25.8), who were separated from their husbands or widowed (24.8, 95\% CI: 21.4-28.5), who were underweight (26.3, 95\% CI: 22.0-31.1), who did not sleep under a mosquito net (21.5, 95\% CI: $19.5-23.6)$, and who were using an intrauterine device (IUD) as a contraceptive method (29.1, 95\% CI: 19.2-41.5). Anemia prevalence was relatively low among those who had completed secondary school (15.9, 95\% CI: 12.1-20.7) and primary school (16.8, 95\% CI: 14.8-19.1), as well as among women in the rich category (16.4, 95\% CI: 14.9-18.0). See Tables 3 and 4.

The bivariate analysis found that 11 variables were significantly associated with anemia: 1 ) province of residence $(p<0.001), 2)$ educational attainment $(p=0.010), 3)$ 
Table 1 Description of socio demographic characteristics of study participants

\begin{tabular}{|c|c|c|}
\hline Variables & & $n$ \\
\hline \multirow[t]{7}{*}{ Age categories } & $15-19$ & 1386 \\
\hline & $20-24$ & 1225 \\
\hline & $25-29$ & 1153 \\
\hline & $30-34$ & 1024 \\
\hline & $35-39$ & 796 \\
\hline & $40-44$ & 614 \\
\hline & $45-49$ & 482 \\
\hline \multirow[t]{5}{*}{ Province } & Kigali city & 899 \\
\hline & South & 1605 \\
\hline & West & 1442 \\
\hline & North & 1088 \\
\hline & East & 1646 \\
\hline \multirow[t]{6}{*}{ Educational attainment } & Complete primary & 1452 \\
\hline & Incomplete primary & 2863 \\
\hline & Incomplete secondary & 1044 \\
\hline & Complete secondary & 340 \\
\hline & Higher & 184 \\
\hline & No education & 797 \\
\hline \multirow[t]{3}{*}{ Economic Status } & Poor & 2622 \\
\hline & Middle class & 1249 \\
\hline & Rich & 2809 \\
\hline \multirow[t]{3}{*}{ Marital status } & Single & 2543 \\
\hline & Married/living together & 3434 \\
\hline & Separated/widowed & 703 \\
\hline \multirow[t]{2}{*}{ Type of residence } & Urban & 1325 \\
\hline & Rural & 5355 \\
\hline
\end{tabular}

economic status $(\mathrm{p}=<0.001), 4)$ marital status $(p=0.001)$, $5)$ pregnancy status $(p=0.019), 6)$ type of FP method used $(p=<0.001), 7)$ nutrition status $(\mathrm{p}<0.001), 8)$ having a mosquito bed net for sleeping ( $p=0.020), 9)$ having slept under a mosquito bed net the night before the survey $(p=0.003), 10)$ type of toilet facility used in the household $(p=0.003)$, and 11$)$ type of residence $(p=0.010)$ See Tables 3 and 4.

\section{Factors associated with anemia}

Six risk factors for anemia among WRA were highlighted in the multivariate analysis: 1) nutrition status, 2) economic status, 3) type of contraceptive method used, 4) use of a mosquito net, 5) marital status, and 6) province of residence (Table 5).

Compared to WRA with normal BMI, WRA who were underweight were 1.39 times as likely to have anemia (OR: 1.39, 95\% CI: 1.09-1.77, $p=0.009$ ) while obese women
Table 2 Description of women' health status and living conditions of study participants

\begin{tabular}{|c|c|c|c|}
\hline Variables & & $n$ & $\%$ \\
\hline \multirow[t]{2}{*}{ Currently pregnant } & No or unsure & 6189 & 92.6 \\
\hline & Yes & 491 & 7.4 \\
\hline \multirow[t]{4}{*}{ Number of children ever born } & Had no child & 2327 & 34.8 \\
\hline & 1-3 children & 2532 & 37.9 \\
\hline & 4-6 children & 1354 & 20.3 \\
\hline & 7 or more & 467 & 7.0 \\
\hline \multirow[t]{2}{*}{ Currently breastfeeding } & No & 4787 & 71.7 \\
\hline & Yes & 1893 & 28.3 \\
\hline \multirow[t]{4}{*}{$\begin{array}{l}\text { Type of contraceptive } \\
\text { method used }\end{array}$} & $\begin{array}{l}\text { None, natural, barriers } \\
\text { or permanent }\end{array}$ & 4572 & 68.4 \\
\hline & Hormonal & 1565 & 23.4 \\
\hline & Intrauterine device (IUD) & 52 & 0.8 \\
\hline & Pregnant & 491 & 7.4 \\
\hline \multirow{4}{*}{$\begin{array}{l}\text { Nutrition status/Body } \\
\text { Mass Index }\end{array}$} & Normal & 4774 & 71.6 \\
\hline & Underweight & 411 & 6.2 \\
\hline & Overweight & 1229 & 18.4 \\
\hline & Obese & 258 & 3.9 \\
\hline \multirow{3}{*}{$\begin{array}{l}\text { Frequency of reading } \\
\text { newspaper or magazine }\end{array}$} & Not at all & 4916 & 73.7 \\
\hline & Less than once a week & 1351 & 20.3 \\
\hline & At least once a week & 403 & 6.0 \\
\hline \multirow{3}{*}{$\begin{array}{l}\text { Frequency of listening } \\
\text { to radio }\end{array}$} & Not at all & 1079 & 16.2 \\
\hline & Less than once a week & 1490 & 22.3 \\
\hline & At least once a week & 4099 & 61.5 \\
\hline \multirow{3}{*}{$\begin{array}{l}\text { Frequency of watching } \\
\text { television }\end{array}$} & Not at all & 3799 & 57.0 \\
\hline & Less than once a week & 1760 & 26.4 \\
\hline & At least once a week & 1107 & 16.6 \\
\hline \multirow{2}{*}{$\begin{array}{l}\text { Have mosquito bed net } \\
\text { for sleeping }\end{array}$} & No & 1049 & 15.7 \\
\hline & Yes & 5631 & 84.3 \\
\hline \multirow{2}{*}{$\begin{array}{l}\text { Respondent slept under } \\
\text { mosquito net }\end{array}$} & No & 2184 & 32.7 \\
\hline & Yes & 4496 & 67.3 \\
\hline \multirow[t]{2}{*}{ Type of toilet facility } & Nonimproved & 1777 & 27.1 \\
\hline & Improved & 4783 & 72.9 \\
\hline \multirow[t]{2}{*}{ Source of drinking water } & Nonimproved & 1677 & 25 \\
\hline & Improved & 4891 & 74.5 \\
\hline \multirow[t]{2}{*}{ Access to health facility/distance } & A big problem & 1428 & 21.4 \\
\hline & Not a big problem & 5252 & \\
\hline
\end{tabular}

were 0.61 times as likely to have anemia (OR: 0.61, 95\% CI: $0.41-0.92, p=0.019)$.

Compared to women in the poor category, women in the rich category were 0.74 times as likely to have anemia (OR: 0.74 , 95\% CI: $0.63-0.87, p<0.001$ ) and women in the middle category were 0.83 times as likely (OR: 0.83 , 95\% CI: $0.69-1.00, p$-value $=0.044$ ) to have anemia. 
Table 3 Prevalence of anemia per socio demographic characteristics

\begin{tabular}{|c|c|c|c|c|c|}
\hline \multirow[t]{2}{*}{ Variables } & & \multicolumn{3}{|c|}{ Anemia prevalence } & \multirow[t]{2}{*}{$P$ value } \\
\hline & & $n$ & $\%$ & $95 \% \mathrm{Cl}$ & \\
\hline \multirow[t]{7}{*}{ Age categories } & $15-19$ & 260 & 18.8 & {$[16.6-21.2]$} & \multirow[t]{7}{*}{0.168} \\
\hline & $20-24$ & 240 & 19.6 & [17.3-22.1] & \\
\hline & $25-29$ & 208 & 18.1 & [15.9-20.5] & \\
\hline & $30-34$ & 184 & 18.0 & [15.6-20.7] & \\
\hline & $35-39$ & 155 & 19.5 & [16.6-22.6] & \\
\hline & $40-44$ & 121 & 19.7 & {$[16.7-23.2]$} & \\
\hline & $45-49$ & 116 & 24.0 & {$[20.3-28.1]$} & \\
\hline \multirow[t]{5}{*}{ Province } & Kigali city & 133 & 14.8 & {$[12.2-17.8]$} & \multirow[t]{5}{*}{$<0.001^{*}$} \\
\hline & South & 367 & 22.9 & {$[20.4-25.6]$} & \\
\hline & West & 258 & 17.9 & [15.6-20.5] & \\
\hline & North & 167 & 15.4 & [13.1-17.9] & \\
\hline & East & 359 & 21.8 & [18.9-25.0] & \\
\hline \multirow[t]{6}{*}{ Educational attainment } & Complete primary & 244 & 16.8 & [14.8-19.1] & \multirow[t]{6}{*}{$0.010^{*}$} \\
\hline & Incomplete primary & 580 & 20.3 & [18.5-22.1] & \\
\hline & Incomplete secondary & 189 & 18.1 & [15.8-20.6] & \\
\hline & Complete secondary & 54 & 15.9 & {$[12.1-20.7]$} & \\
\hline & Higher & 38 & 20.7 & [15.6-26.9] & \\
\hline & No education & 179 & 22.5 & [19.5-25.8] & \\
\hline \multirow[t]{3}{*}{ Economic Status } & Poor & 589 & 22.5 & {$[20.6-24.5]$} & \multirow[t]{3}{*}{$<0.001 *$} \\
\hline & Middle class & 235 & 18.8 & {$[16.3-21.7]$} & \\
\hline & Rich & 460 & 16.4 & [14.9-18.0] & \\
\hline \multirow[t]{3}{*}{ Marital status } & Single & 485 & 19.1 & [17.4-20.8] & \multirow[t]{3}{*}{$0.001 *$} \\
\hline & Married/living together & 625 & 18.2 & [16.7-19.8] & \\
\hline & Separated/widowed & 174 & 24.8 & {$[21.4-28.5]$} & \\
\hline \multirow[t]{2}{*}{ Type of residence } & Urban & 217 & 16.3 & {$[14.3-18.6]$} & \multirow[t]{2}{*}{$0.010^{*}$} \\
\hline & Rural & 1068 & 19.9 & {$[18.5-21.5]$} & \\
\hline
\end{tabular}

$P$ value are from Pearson's chi-squared test

*: Unadjusted significant variables $(p<0.05)$

Women who were using hormonal contraceptives were 0.6 times as likely to have anemia (OR: 0.60, 95\% CI: $0.50-0.72$, p-value: $<0.001)$ while those who were using intrauterine devices were 1.97 times as likely to have anemia (OR: 1.97, 95\% CI: 1.04-3.73, p-value = 0.037) compared with those using natural barriers, permanent contraceptive methods, or no contraceptive method. Compared to women who were not married, women who were separated or widowed were 1.35 times as likely to have anemia (OR: $1.35,95 \%$ CI: $1.09-1.67$, $p=0.006)$. There was no difference between married and non-married women $(p=0.25)$.

Women who reported sleeping under a mosquito net the night before the survey were 0.85 times as likely to have anemia (OR: $0.85,95 \%$ CI: $0.73-0.98, p=0.025$ ) than those who did not sleep under a mosquito net the night before the survey. Additionally, women who were living in Southern province (OR: 1.45, 95\% CI: 1.15-
1.82, $p=0.002)$ and in Eastern province (OR: 1.41, 95\% CI: $1.11-1.79, p=0.005)$ were 1.45 and 1.41 times as likely to have anemia, respectively compared to WRA living in Kigali city.

\section{Discussion}

The objectives of this study were to identify the describes of the background characteristics of WRA in Rwanda with anemia and to therefore identify associated risk factors. According to the analysis, the prevalence of anemia among WRA in Rwanda was $19.2 \%$. Although the anemia prevalence in Rwanda was lower than many other countries in the Sub-Saharan Africa region [2, 9], it is still considered a public health problem according to WHO criteria [23]. In addition, the prevalence increased between 2010 and 2015 and varied across population subgroups $[10,24]$. In this study, it was found to vary with age, province of residence, education level, 
Table 4 Prevalence of anemia per women' health status and living conditions

\begin{tabular}{|c|c|c|c|c|c|}
\hline \multirow[t]{2}{*}{ Variables } & & \multicolumn{3}{|c|}{ Anemia prevalence } & \multirow[t]{2}{*}{$P$ value } \\
\hline & & $n$ & $\%$ & $95 \% \mathrm{Cl}$ & \\
\hline \multirow[t]{2}{*}{ Currently pregnant } & No or unsure & 1170 & 18.9 & {$[17.7-20.2]$} & $0.019^{*}$ \\
\hline & Yes & 115 & 23.4 & {$[19.6-27.6]$} & \\
\hline \multirow[t]{4}{*}{ Number of children ever born } & Had no child & 456 & 19.6 & {$[17.9-21.4]$} & 0.684 \\
\hline & 1-3 children & 472 & 18.6 & {$[17.0-20.4]$} & \\
\hline & 4-6 children & 259 & 19.2 & {$[17.0-21.6]$} & \\
\hline & 7 or more & 97 & 20.8 & {$[17.1-25.2]$} & \\
\hline \multirow[t]{2}{*}{ Currently breastfeeding } & No & 919 & 19.2 & {$[17.8-20.6]$} & 0.918 \\
\hline & Yes & 366 & 19.3 & {$[17.4-21.4]$} & \\
\hline \multirow[t]{4}{*}{ Type of contraceptive method used } & None, natural, barriers or permanent & 940 & 20.6 & {$[19.1-22.1]$} & $<0.001 *$ \\
\hline & Hormonal & 215 & 13.7 & {$[11.9-15.8]$} & \\
\hline & Intrauterine device (IUD) & 15 & 29.1 & {$[19.2-41.5]$} & \\
\hline & Pregnant & 115 & 23.4 & {$[19.6-27.6]$} & \\
\hline \multirow[t]{4}{*}{ Nutrition status/Body Mass Index } & Normal & 912 & 19.1 & {$[17.8-20.5]$} & $<0.001^{*}$ \\
\hline & Underweight & 108 & 26.3 & {$[22.0-31.1]$} & \\
\hline & Overweight & 233 & 19.0 & {$[16.7-21.5]$} & \\
\hline & Obese & 30 & 11.6 & {$[8.1-16.4]$} & \\
\hline \multirow[t]{3}{*}{ Frequency of reading newspaper or magazine } & Not at all & 970 & 19.7 & {$[18.3-21.2]$} & 0.199 \\
\hline & Less than once a week & 235 & 17.4 & {$[15.4-19.6]$} & \\
\hline & At least once a week & 78 & 19.3 & {$[15.5-23.8]$} & \\
\hline \multirow[t]{3}{*}{ Frequency of listening to radio } & Not at all & 233 & 21.6 & {$[18.8-24.7]$} & 0.138 \\
\hline & Less than once a week & 292 & 19.6 & {$[17.2-22.1]$} & \\
\hline & At least once a week & 759 & 18.5 & {$[17.1-20.0]$} & \\
\hline \multirow[t]{3}{*}{ Frequency of watching television } & Not at all & 755 & 19.9 & {$[18.3-21.5]$} & 0.237 \\
\hline & Less than once a week & 330 & 18.8 & {$[16.8-20.9]$} & \\
\hline & At least once a week & 195 & 17.6 & {$[15.3-20.1]$} & \\
\hline \multirow[t]{2}{*}{ Have mosquito bed net for sleeping } & No & 232 & 22.1 & {$[19.4-25.1]$} & $0.020^{*}$ \\
\hline & Yes & 1052 & 18.7 & {$[17.4-20.1]$} & \\
\hline \multirow[t]{2}{*}{ Respondent slept under mosquito net } & No & 470 & 21.5 & {$[19.5-23.6]$} & $0.003^{*}$ \\
\hline & Yes & 815 & 18.1 & {$[16.8-19.6]$} & \\
\hline \multirow[t]{2}{*}{ Type of toilet facility } & Nonimproved & 391 & 22.0 & {$[19.7-24.4]$} & $0.003^{*}$ \\
\hline & Improved & 871 & 18.2 & [16.9-19.6] & \\
\hline \multirow[t]{2}{*}{ Source of drinking water } & Nonimproved & 327 & 19.5 & {$[17.0-22.3]$} & 0.785 \\
\hline & Improved & 935 & 19.1 & {$[17.8-20.5]$} & \\
\hline \multirow[t]{2}{*}{ Access to health facility/ distance } & A big problem & 285 & 20.0 & {$[17.5-22.7]$} & 0.503 \\
\hline & Not a big problem & 999 & 19.0 & {$[17.7-20.4]$} & \\
\hline
\end{tabular}

$P$ values are from Pearson's chi-squared test

*: Unadjusted significant variables $(p<0.05)$

marital status, the type of contraceptive method used as well as the economic and nutrition status. Our study showed that women who were obese or rich, as well as those who slept under a mosquito net or used hormonal contraceptives were less likely to have anemia while those who were underweight, used intrauterine devices as a contraceptive method, and lived in the Southern or
Eastern provinces were more likely to have anemia, than were individuals in their respective comparison groups.

Similar to studies in other settings including Ethiopia and Pakistan, our analysis found that poor and undernourished women were more likely to have anemia [25, 26]. Anemia is a multifaceted problem where nutrition and economic status work in synergy. Evidence suggests 
Table 5 Factors associated with anemia: multivariate analysis results (Logistic Regression)

\begin{tabular}{|c|c|c|c|c|c|c|}
\hline \multirow[t]{2}{*}{ Variables } & \multicolumn{3}{|c|}{ Full model } & \multicolumn{3}{|c|}{ Final model } \\
\hline & $\overline{\mathrm{AOR}}$ & $95 \% \mathrm{Cl}$ & $\overline{P \text {-value }}$ & $\overline{\mathrm{AOR}}$ & $95 \% \mathrm{Cl}$ & $P$ P-value \\
\hline \multicolumn{7}{|l|}{ Age categories } \\
\hline $15-19$ & 1 & & & & & \\
\hline $20-24$ & 1.13 & {$[0.90-1.42]$} & 0.285 & & & \\
\hline $25-29$ & 1.01 & {$[0.77-1.33]$} & 0.915 & & & \\
\hline $30-34$ & 1.02 & {$[0.76-1.37]$} & 0.871 & & & \\
\hline $35-39$ & 1.18 & {$[0.86-1.60]$} & 0.304 & & & \\
\hline $40-44$ & 1.07 & {$[0.77-1.49]$} & 0.669 & & & \\
\hline $45-49$ & 1.24 & {$[0.88-1.75]$} & 0.223 & & & \\
\hline \multicolumn{7}{|l|}{ Nutrition status/Body Mass Index } \\
\hline Normal & 1 & & & 1 & & \\
\hline Underweight & $1.38^{*}$ & {$[1.08-1.78]$} & 0.011 & $1.39^{*}$ & {$[1.09-1.77]$} & 0.009 \\
\hline Overweight & 1.1 & {$[0.93-1.31]$} & 0.270 & 1.09 & {$[0.92-1.30]$} & 0.301 \\
\hline Obese & $0.60^{*}$ & {$[0.40-0.91]$} & 0.015 & $0.61^{*}$ & {$[0.41-0.92]$} & 0.019 \\
\hline \multicolumn{7}{|l|}{ Economic Status } \\
\hline Poor & 1 & & & 1 & & \\
\hline Middle class & 0.83 & {$[0.69-1.01]$} & 0.057 & $0.83^{*}$ & {$[0.69-1.00]$} & 0.044 \\
\hline Rich & $0.74^{* *}$ & {$[0.61-0.89]$} & 0.002 & $0.74^{*}$ & {$[0.63-0.87]$} & $<0.001$ \\
\hline \multicolumn{7}{|l|}{ Type of contraceptive method used } \\
\hline None - natural - barriers or permanent & 1 & & & 1 & & \\
\hline Hormonal & $0.60^{*}$ & {$[0.49-0.73]$} & $<0.001$ & $0.60^{*}$ & {$[0.50-0.72]$} & $<0.001$ \\
\hline IUD & $1.93^{*}$ & {$[1.01-3.68]$} & 0.045 & $1.97^{*}$ & {$[1.04-3.73]$} & 0.037 \\
\hline Pregnant & 1.18 & {$[0.90-1.53]$} & 0.227 & 1.15 & {$[0.89-1.49]$} & 0.275 \\
\hline \multicolumn{7}{|l|}{ Educational attainment } \\
\hline No education & 1 & & & & & \\
\hline Incomplete primary & 0.97 & {$[0.78-1.21]$} & 0.810 & & & \\
\hline Complete primary & 0.81 & {$[0.64-1.03]$} & 0.087 & & & \\
\hline Incomplete secondary & 0.99 & {$[0.74-1.32]$} & 0.929 & & & \\
\hline Complete secondary & 0.89 & {$[0.61-1.30]$} & 0.545 & & & \\
\hline Higher & 1.35 & {$[0.88-2.08]$} & 0.171 & & & \\
\hline \multicolumn{7}{|l|}{ Respondent slept under mosquito bed net } \\
\hline No & 1 & & & 1 & & \\
\hline Yes & $0.85^{*}$ & {$[0.74-0.98]$} & 0.031 & $0.85^{*}$ & {$[0.73-0.98]$} & 0.025 \\
\hline \multicolumn{7}{|l|}{ Marital status } \\
\hline Single & 1 & & & 1 & & \\
\hline Married/living together & 1.07 & {$[0.86-1.34]$} & 0.537 & 1.11 & {$[0.94-1.30]$} & 0.215 \\
\hline Separated/widowed & 1.28 & {$[0.98-1.69]$} & 0.073 & $1.35^{*}$ & {$[1.09-1.67]$} & 0.006 \\
\hline \multicolumn{7}{|l|}{ Type of toilet facility } \\
\hline Nonimproved & 1 & & & & & \\
\hline Improved & 0.96 & {$[0.82-1.12]$} & 0.624 & & & \\
\hline \multicolumn{7}{|l|}{ Type of residence } \\
\hline Urban & 1 & & & & & \\
\hline Rural & 0.97 & {$[0.79-1.18]$} & 0.745 & & & \\
\hline
\end{tabular}


Table 5 Factors associated with anemia: multivariate analysis results (Logistic Regression) (Continued)

\begin{tabular}{|c|c|c|c|c|c|c|}
\hline \multirow[t]{2}{*}{ Variables } & \multicolumn{3}{|c|}{ Full model } & \multicolumn{3}{|c|}{ Final model } \\
\hline & $\overline{A O R}$ & $95 \% \mathrm{Cl}$ & $\overline{\text { P-value }}$ & $\mathrm{AOR}$ & $95 \% \mathrm{Cl}$ & P-value \\
\hline \multicolumn{7}{|c|}{ Province of residence } \\
\hline Kigali city & 1 & & & 1 & & \\
\hline South & $1.52^{*}$ & [1.17-1.97] & 0.002 & $1.45^{*}$ & {$[1.15-1.82]$} & 0.002 \\
\hline West & 1.07 & {$[0.81-1.41]$} & 0.636 & 1.03 & {$[0.80-1.33]$} & 0.814 \\
\hline North & 0.95 & {$[0.71-1.28]$} & 0.756 & 0.92 & {$[0.70-1.20]$} & 0.533 \\
\hline East & $1.50^{*}$ & {$[1.15-1.95]$} & 0.003 & $1.41^{*}$ & {$[1.11-1.79]$} & 0.005 \\
\hline
\end{tabular}

*: Adjusted significant variables $(p<0.05)$ from multiple logistic regression AOR: Adjusted Odds Ratio.

that improved economic status is associated with appropriate nutrition conditions [27], lower infection morbidity [26], increased access to health services as well as other favourable living conditions [27, 28], all of which in turn influence anemia. Malnourished women have greater risk of iron deficiency, the most common proximate cause of anemia [1] and malnutrition is often associated with poor socio-economic status [29]. Interventions that aim to empower women economically should be considered in order to reduce anemia prevalence. Moreover, malnutrition management programs should ensure that iron supplementation is sustained within intervention packages.

In this study, the use of hormonal contraceptives was associated with lower risk of anemia among WRA, while the use of IUD was associated with higher risk. Similar findings were seen in other studies conducted in 14 different low- and middle-income countries including Tanzania and Ethiopia [25, 30,31]. Another study conducted in seven countries also found that hormonal contraceptive users had higher haemoglobin and ferritin levels compared to non-users [32]. Using hormonal contraceptive can be resulted in less bleeding during the menstruation, which ultimately reduces blood loss over time [33, 34].

A study conducted in Pakistan also observed higher anemia risk among IUD users [35]. IUD may increase uterine blood flow as well as volume of bleeding during and duration of menstruation periods, especially during the first months of usage, which in turn increase the likelihood of anemia [36, 37]. In addition, some research has found that IUD users have a reduction in hemoglobin content and iron saturation/ ferritin levels, which may trigger or worsen existing anemia [32, 38]. While more investigations are needed to understand the real physiological mechanisms, our study findings supported the existing evidence that IUD use is among the risk factors of anemia in WRA. Clinical guidelines should consider specifying treatments for IUD-induced bleeding [39] as well recommending iron supplementation for IUD users especially during the first months of usage.
Geographic area of residence was found to be associated with anemia, with women in the Eastern and Southern provinces being more likely to have anemia. The Eastern and Southern provinces in Rwanda are considered to be high malaria endemic regions; higher risk of malaria also translates to higher anemia risk [40]. Similar associations between anemia and geographic location have been found in Tanzania [30]. In Rwanda, iron supplementation during pregnancy is less common in the Eastern province than in other provinces [10]. Interventions should consider including iron supplementation, promotion of foods rich in iron and other micronutrients, as well as to prevent malaria [12, 41]. The most affected geographic areas should be prioritized.

Consistent with the results from other studies, sleeping under mosquito nets was associated with lower likelihood of anemia in our study, which makes sense given that malaria itself is a risk factor of anemia [42]. As mosquito net coverage and usage remain challenges in many developing countries [43-45], malaria prevention strategies including efforts to ensure the availability as well as proper use of mosquito nets in the community should be integrated in anemia prevention programs.

Widowers or women separated from their husbands were more likely to have anemia. Traditionally men are breadwinners in many developing societies [46]. Widows and women separated from their husbands lack support to sustain their families, predisposing them to economic deprivation, poverty, malnutrition and low access to health services [27-29]. In addition, our analysis showed a correlation between marital status and age $(r=0.63)$. Older age was found to be associated with anemia in some studies [25]. While further investigations are needed to better understand the possible associations between marital status, age and anemia status, our findings suggest that old women, especially widows, may face many other health problems that are understudied. Special attention and priority should be given to understanding the health needs of this vulnerable group.

Our study found that women with lower education levels had slightly higher prevalence of anemia, although 
statistical significance was not found. Other studies have found education level to be a risk factor for anemia [25, 30]. The differences in settings of the studies could be related to the discrepancy. In the 2014/2015 Rwanda DHS, only $19 \%$ of women had no education; while $67 \%$ were reported in Ethiopia in 2005 and 27\% in Tanzania in $2010[10,47,48]$, the variation in the sample composition could have affected the analysis outcome.

Interestingly, this study found that about $40 \%$ of the women classified as "rich" also had anemia. This result was inconsistent with other evidence. Despite economic improvement in Rwanda over recent decades, about 39\% of the population remained living under poverty line [49, 50]. Further investigation found that DHS, the Ministry of Local Government and Social Affairs in Rwanda used different socioeconomic classification methodologies. The DHS used five categories: poorest, poor, middle, rich and richest, based on the durable goods owned by a household such as television, mobile telephone and other household characteristics such as access to electricity and source of drinking water [10], while the Rwanda Ministry of Local Government and Social Affairs uses four categories for social stratification: very poor, poor, middle and rich [51, 52]. In recent years, there has been considerable debate over whether the stratification systems truly reflect the economic status of the population $[53,54]$. Acknowledging that the limitations of the stratifications may cause discrepancies, the Rwanda government is currently revising the categorization to reflect the true population economic status [52].

This study successfully identified some risk factors among WRA in Rwanda and proposed some recommendations. However, the results must be seen in light of some limitations. This study could only use variables that were in the DHS, due to the nature of secondary data analysis. Qualitative information could provide increased understanding of the attitudes and practices related to variations in food consumption patterns. However, the DHS survey used a national representative sample and was conducted with standardized quality assurance measures in both data collection and management to ensure reliability and validity of the results [55, $56]$, which could improve the generalizability of the results of our analysis.

\section{Conclusions}

In order to address anemia among WRA in Rwanda, programs should seek to improve women's economic livelihoods and nutritional statuses. Furthermore, clinical guidelines should ensure that IUD users have access IUDrelated bleeding treatments as well as iron supplements. Special attention should be provided based on geographic variations. Integrating malaria prevention strategies into anemia program should also be considered.

\section{Abbreviations}

AOR: Adjusted Odds Ratio; Cl: Confidence Interval; DHS: Demographic and Health Survey; FP: Family Planning; Hb: Hemoglobin; OR: Odds Ratio; RDHS: Rwanda Demographic and Health Survey; WHO: World Health Organization; WRA: Women of Reproductive Age

\section{Acknowledgements}

The authors thank Dr. Michael Law, Associate Professor from the University of British Columbia - School of Population and Public Health and Dr. Vedaste Ndahindwa, Lecturer from the University of Rwanda - School of Public Health, for their guidance and support on this study. In addition, the authors thank the faculty from the University of Rwanda for their support during the courses. Furthermore, the authors also thank the MEASURE DHS Project for availing open access to the data.

\section{Authors' contributions}

$\mathrm{DH}$ designed the project, acquired the data, conducted the literature review, wrote the manuscript, and was responsible to submit it. DH and MN conducted the statistical analysis. RW and $J$ provided overall advisory on the project and manuscript revision. All authors reviewed and approved the final manuscript.

Funding

No funding received for this study.

\section{Availability of data and materials}

The data used for this study are from the Rwanda Demographic and Health surveys (DHS) and are publicly available here: https://dhsprogram.com/data/ available-datasets.cfm

\section{Ethics approval and consent to participate}

Since this study was a secondary analysis of the Rwanda Demographic and Health surveys (RDHS) data, which are publicly available, the study did not require any ethics approval. Only DHS program authorization was requested to download the dataset.

\section{Consent for publication}

Being a secondary analysis, no consent to publish was needed for this study. There was no identifiable data.

\section{Competing interests}

No competing interests by the authors.

\section{Author details}

${ }^{1}$ Department of Global Health Delivery, University of Global Health Equity, Kigali, Rwanda. ${ }^{2}$ School of Population and Public Health, University of British Columbia, Vancouver, BC, Canada. ${ }^{3}$ School of Public Health, Yale University, New Haven, CT, USA.

Received: 16 September 2019 Accepted: 29 November 2019

Published online: 11 December 2019

\section{References}

1. Kassebaum N on behalf of G 2013 AC. The global burden of Anemia. Hematol Oncol Clin North Am [Internet]. 2016;30(2):247-308. https://doi.org/ 10.1016/j.hoc.2015.11.002.

2. World Health Organization. The global prevalence of Anaemia in 2011. WHO Rep [Internet]. 2015;48. Available from: http://apps.who.int/iris/ bitstream/10665/177094/1/9789241564960_eng.pdf?ua=1

3. Daru J, Zamora J, Fernández-Félix BM, Vogel J, Oladapo OT, Morisaki N, et al. Risk of maternal mortality in women with severe anaemia during pregnancy and post partum: a multilevel analysis. Lancet Glob Heal. 2018;6(5):e548-54.

4. Frass KA. Postpartum hemorrhage is related to the hemoglobin levels at labor: observational study. Alexandria J Med. 2015;51(4):333-7.

5. Rahman MM, Abe SK, Rahman MS, Kanda M, Narita S, Bilano V, et al. Maternal anemia and risk of adverse birth and health outcomes in low- and middle-income countries: systematic review and meta-analysis. Am J Clin Nutr [Internet]. 2016;103(2):495-504. Available from: http://www.ncbi.nlm. nih.gov/pubmed/26739036. 
6. Ntenda PAM, Nkoka O, Bass P, Senghore T. Maternal anemia is a potential risk factor for anemia in children aged 6-59 months in Southern Africa: a multilevel analysis. BMC Public Health. 2018;18:1-13.

7. Haas JD, Brownlie T. Iron deficiency and reduced work capacity: a critical review of the research to determine a causal relationship. J Nutr [Internet]. 2001;131(2S-2):676S-88S; discussion 688S-690S. Available from: http://www. ncbi.nlm.nih.gov/pubmed/11160598.

8. Smith RE. The clinical and economic burden of anemia. Am J Manag Care [Internet]. 2010;16(Suppl I):S59-66. Available from: http://www.ncbi.nlm.nih. gov/pubmed/20297873.

9. Stevens GA, Finucane MM, De-Regil LM, Paciorek CJ, Flaxman SR, Branca F, et al. Global, regional, and national trends in haemoglobin concentration and prevalence of total and severe anaemia in children and pregnant and non-pregnant women for 1995-2011: A systematic analysis of populationrepresentative data. Lancet Glob Health. 2013;1(1):e16-25.

10. National Institute of Statistics of Rwanda (NISR), Ministry of Health (MOH) [Rwanda] and II. Rwanda Demographic and Health Survey 2014-15 Final Report. Rockville: NISR, MOH, and ICF International; 2015.

11. McLean E, Cogswell M, Egli I, Wojdyla D, de Benoist B. Worldwide prevalence of anaemia, WHO vitamin and mineral nutrition information system, 19932005. Public Health Nutr [Internet]. 2009;12(4):444-54. Available from: http:// whqlibdoc.who.int/publications/2008/9789241596657_eng.pdf

12. Haas JD, Luna SV, Lung'aho MG, Wenger MJ, Murray-Kolb LE, Beebe S, et al. Consuming iron biofortified beans increases iron status in Rwandan Women after 128 days in a randomized controlled feeding trial. J Nutr [Internet]. 2016;146(8):1586-92. https://doi.org/10.3945/jn.115.224741.

13. Masaisa F, Gahutu JB, Mukiibi J, Delanghe J, Philippé J. Anemia in human immunodeficiency virus-infected and uninfected women in Rwanda. Am J Trop Med Hyg. 2011;84(3):456-60.

14. Kateera F, Ingabire CM, Hakizimana E, Kalinda P, Mens PF, Grobusch MP, et al. Malaria, anaemia and under-nutrition: Three frequently co-existing conditions among preschool children in rural Rwanda. Malar J. 2015; 14(1):art440.

15. Danquah I, Gahutu JB, Zeile I, Musemakweri A, Mockenhaupt FP. Anaemia, iron deficiency and a common polymorphism of iron-regulation, TMPRSS6 rs855791, in rwandan children. Trop Med Int Heal. 2014;19(1):117-22.

16. Donahue Angel M, Berti P, Siekmans K, Tugirimana PL, Boy E. Prevalence of Iron deficiency and Iron deficiency Anemia in the northern and southern provinces of Rwanda. Food Nutr Bull. 2017;38(4):554-63.

17. Murray-Kolb LE, Wenger MJ, Scott SP, Rhoten SE, Lung'aho MG, Haas JD. Consumption of iron-biofortified beans positively affects cognitive performance in 18- to 27-Year-Old Rwandan Female College Students in an 18-Week randomized controlled efficacy trial. J Nutr [Internet]. 2017 [cited 2019 Aug 30];in255356; https://doi.org/10.3945/jn.117.255356.

18. Munyazesa E, Emile I, Mutimura E, Hoover DR, Shi Q, McGinn AP, et al. Assessment of haematological parameters in HIV-infected and uninfected Rwandan women: a cross-sectional study. BMJ Open. 2012;2(6):e001600.

19. National Institute of Statistics of Rwanda. Fourth Population and Housing Census, Rwanda, 2012 Final Results, Main indicators report [Internet]. Kigali; 2014. Available from: http://www.Imis.gov.rw/scripts/publication/reports/ FourthRwandaPopulationandHousingCensus_Housing.pdf

20. The World Bank Group. GDP per capita (current US\$) | Data [Internet]. 2017. 2017 [cited 2019 Jan 9]. Available from: https://data.worldbank.org/ indicator/NY.GDP.PCAP.CD

21. Binagwaho A, Farmer PE, Nsanzimana S, Karema C, Gasana M, de Dieu Ngirabega J, et al. Rwanda 20 years on: investing in life. Lancet [Internet]. 2014;384(9940):371-5. Available from: http://www.ncbi.nlm.nih.gov/pmc/ articles/PMC4151975/

22. WHO. Nutrition - Body mass index - BMI [Internet]. World Health Organization. 2019 [cited 2019 Nov 6]. Available from: http://www.euro.who. int/en/health-topics/disease-prevention/nutrition/a-healthy-lifestyle/bodymass-index-bmi

23. World Health Organization. Worldwide prevalence of anaemia 1993-2005. WHO global database on Anaemia [internet]. World Health Organization. 2008. Available from: http://whqlibdoc.who.int/publications/2008/97892415 96657_eng.pdf?ua=1

24. NISR - MOH and ICF International. Rwanda Demographic and Health Survey 2010. 2011.

25. Gebremedhin S, Enquselassie F. Correlates of anemia among women of reproductive age in Ethiopia: evidence from Ethiopian DHS 2005. Ethiop J Heal Dev. 2011;25(1):22-30.
26. Soofi S, Khan GN, Sadiq K, Ariff S, Habib A, Kureishy S, et al. Prevalence and possible factors associated with anaemia, and vitamin $B_{12}$ and folate deficiencies in women of reproductive age in Pakistan: analysis of nationallevel secondary survey data. BMJ Open [Internet]. 2017;7(12):e018007. https://doi.org/10.1136/bmjopen-2017-018007.

27. Apouey $\mathrm{BH}$. Health policies and the relationships between socioeconomic status, access to health care, and health. Isr J Health Policy Res. 2013;2(1):1-2.

28. Arpey NC, Gaglioti AH, Rosenbaum ME. How socioeconomic status affects patient perceptions of health care: a qualitative study. J Prim Care Community Health. 2017;8:169.

29. Mawani M, Aziz AS. Iron deficiency Anemia among women of reproductive age, an important public health problem: situation analysis. Reprod Syst Sex Disord [Internet]. 2016;5(3):1-6. Available from: https://www.omicsonline. org/open-access/iron-deficiency-anemia-among-women-of-reproductiveage-an-important-public-health-problem-situation-analysis-2161-038X-10001 87.php?aid $=78570$

30. Wilunda C, Massawe S, Jackson C. Determinants of moderate-to-severe anaemia among women of reproductive age in Tanzania: analysis of data from the 2010 Tanzania demographic and health survey. Trop Med Int Heal. 2013;18(12):1488-97.

31. Bellizzi S, Ali MM. Effect of oral contraception on anemia in 12 low- and middle-income countries. Contraception [Internet]. 2018;97(3):236-42. https://doi.org/10.1016/j.contraception.2017.11.001.

32. Bathija H, Lei ZW, Cheng XQ, Xie L, Wang Y, Rugpao S, et al. Effects of contraceptives on hemoglobin and ferritin. Contraception. 1998;58:261.

33. Hillard PA. Menstrual suppression: current perspectives. Int J Women's Health. 2014;6(1):631-7.

34. Miller L, Hughes JP. Continuous combination oral contraceptive pills to eliminate withdrawal bleeding: a randomized trial. Obstet Gynecol [Internet]. 2003;101(4):653-61. [cited 2018 Jul 8] Available from: http://www. ncbi.nlm.nih.gov/pubmed/12681866.

35. Khan R, Jamil S. Hematologic variations associated with the long term use of contraceptives in young females. Am J Phytomed Clin Ther. 2014;2:580-6.

36. Fouda UM, Yossef D, Gaafar HM. Uterine artery blood flow in patients with copper intrauterine device-induced abnormal uterine bleeding. Middle East Fertil Soc J [Internet]. 2010;15(3):168-73. https://doi.org/10.1016/j.mefs.2010.07.003.

37. Turok DK, Gawron L, Steele K, Storck KBH. Tracking IUD Bleeding Experiences (TRIBE): A prospective evaluation of bleeding profiles among new IUD users. Contraception. 2016;94:407.

38. Hassan EO, El-Husseini M, El-Nahal N. The effect of 1-year use of the Cu-T 380a and oral contraceptive pills on hemoglobin and ferritin levels. Contraception. 1999;60:101.

39. Godfrey EM, Folger SG, Jeng G, Jamieson DJ, Curtis KM. Treatment of bleeding irregularities in women with copper-containing IUDs: a systematic review. Contraception. 2013;87:549.

40. Castelli F, Sulis F, Caligaris $S$. The relationship between anaemia and malaria: apparently simple, yet controversial. Trans R Soc Trop Med Hyg. 2014;108(4):181-2.

41. Wang $Z$, Sun J, Wang $L$, Zong $M$, Chen $Y$, Lin $Y$, et al. [Effect of iron supplementation on iron deficiency anemia of childbearing age women in Shanghai]. Wei Sheng Yan Jiu [Internet]. 2012. [cited 2019 Aug 22];41(1):515. Available from: http://www.ncbi.nlm.nih.gov/pubmed/22443058.

42. Ouma P, Van Eijk AM, Hamel MJ, Parise M, Ayisi JG, Otieno K, et al. Malaria and anaemia among pregnant women at first antenatal clinic visit in Kisumu, western Kenya. Trop Med Int Heal. 2007;12(12):1515-23.

43. Larsen DA, Hutchinson P, Bennett A, Yukich J, Anglewicz P, Keating J, et al. Community coverage with insecticide-treated mosquito nets and observed associations with all-cause child mortality and malaria parasite infections. Am J Trop Med Hyg. 2014;91(5):950-8.

44. Ntonifor $\mathrm{NH}$, Veyufambom $\mathrm{S}$. Assessing the effective use of mosquito nets in the prevention of malaria in some parts of Mezam division, Northwest Region Cameroon. Malar J. 2016;15(1):1-8.

45. Korenromp EL, Miller J, Cibulskis RE, Kabir CM, Alnwick D, Dye C. Monitoring mosquito net coverage for malaria control in Africa: possession vs. use by children under 5 years. Trop Med Int Health. 2003;8(8):693-703.

46. Sossou MA. Widowhood practices in West Africa: the silent victims. Int J Soc Welf. 2002;11(3):201-9.

47. National Bureau of Statistics (NBS) [Tanzania] and ICF Macro. Tanzania Demographic and Health Survey 2016 [Internet]. Dar es Salaam, Tanzania: NBS and ICF Macro. 2011. Available from: http://www.measuredhs.com/ pubs/pdf/FR243/FR243\%5B24June2011\%5D.pdf 
48. Central Statistical Agency [Ethiopia] and ORC Macro. Ethiopia Demographic and Health Survey 2005 [Internet]. Addis Ababa, Ethiopia and Calverton, Maryland, USA: Central Statistical Agency and ORC Macro. 2006. Available from: http://www.measuredhs.com/pubs/pdf/FR179/FR179[23June201 1].pdf.

49. National Institute of Statistics of Rwanda (NISR). The Fifth Integrated Household Living Conditions Survey. ElCV5 2016/17: Rwanda Poverty Profile Report. 2018.

50. The World Bank Group. Rwanda Overview [Internet]. The World Bank Group. 2019 [cited 2019 Nov 7]. Available from: https://www.worldbank.org/en/ country/rwanda/overview

51. Gouvement of Rwanda. Community-led Ubudehe categorisation kicks off [Internet]. Gouvement of Rwanda. 2015 [cited 2019 Nov 7]. Available from: http://www.gov.rw/news detail/?tx ttnews\%5Btt_news\%5D=1054\&cHash= a315a8b0054e76f9c699f05ce24d3eb8

52. Ministry of Local Government (MINALOC) Rwanda. MINALOC launches national consultations on ubudehe categorisation review [Internet]. Ministry of Local Government (MINALOC) Rwanda. 2019 [cited 2019 Nov 6]. Available from: http://197.243.22.137/minaloc/index.php?id=469\&tx_news _

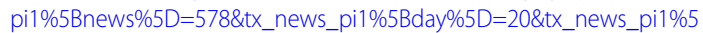
Bmonth\%5D=2\&tx_news_pi1\%5Byear\%5D=2019\&cHash=d1836c2f1 1e8e2 dcb3e3e7ca2ed22f37

53. Ezeanya-Esiobu C. The Rise of homegrown ideas and grassroots voices new directions in social policy in Rwanda prepared for the UNRISD project on New Directions in Social Policy: alternatives for and from the Global South [Internet]. 2017 [cited 2019 Nov 6]. Available from: www.unrisd.org

54. Rwanda Governance Board. Annual Report 2017-2018 and Imihigo 20182019 [Internet]. KIGALI- Rwanda; 2018. Available from: http://www.rgb.rw/ fileadmin/Publications/Annual_report/RGB_Annual_Report_2017-2018.pdf

55. Corsi DJ, Neuman M, Finlay JE, Subramanian SV. Demographic and health surveys: a profile. Int J Epidemiol. 2012;41:1602.

56. ICF International. Demographic and health surveys sampling and household listing manual. Calverton: MEASURE DHS; 2012.

\section{Publisher's Note}

Springer Nature remains neutral with regard to jurisdictional claims in published maps and institutional affiliations.

Ready to submit your research? Choose BMC and benefit from:

- fast, convenient online submission

- thorough peer review by experienced researchers in your field

- rapid publication on acceptance

- support for research data, including large and complex data types

- gold Open Access which fosters wider collaboration and increased citations

- maximum visibility for your research: over $100 \mathrm{M}$ website views per year

At $\mathrm{BMC}$, research is always in progress.

Learn more biomedcentral.com/submissions 